\title{
A Configurable Component-Based Software System for Magnetic Field Measurements
}

\author{
J. M. Nogiec, J. DiMarco, S. Kotelnikov, K. Trombly-Freytag, D. Walbridge, and M. Tartaglia
}

\begin{abstract}
A new software system to test accelerator magnets has been developed at Fermilab. The magnetic measurement technique involved employs a single stretched wire to measure alignment parameters and magnetic field strength. The software for the system is built on top of a flexible component-based framework, which allows for easy reconfiguration and runtime modification. Various user interface, data acquisition, analysis, and data persistence components can be configured to form different measurement systems that are tailored to specific requirements (e.g., involving magnet type or test stand). The system can also be configured with various measurement sequences or tests, each of them controlled by a dedicated script. It is capable of working interactively as well as executing a preselected sequence of tests. Each test can be parameterized to fit the specific magnet type or test stand requirements. The system has been designed with portability in mind and is capable of working on various platforms, such as Linux, Solaris, and Windows. It can be configured to use a local data acquisition subsystem or a remote data acquisition computer, such as a VME processor running VxWorks. All hardware-oriented components have been developed with a simulation option that allows for running and testing measurements in the absence of data acquisition hardware.
\end{abstract}

Index Terms - Superconducting device measurements, accelerator measurement systems, computer applications, reconfigurable architectures.

\section{INTRODUCTION}

$\mathrm{T}$ HOROUGH testing of superconducting accelerator magnets requires a variety of tests employing various hardware setups and procedures. This in turn requires many measurement systems, frequently of quite different organization and technology. At Fermilab an effort was launched to build all measurement systems as a family of systems based on a common software framework. Such an approach has several advantages over the typical nonintegrated solutions, including decreased maintenance efforts, shortened development time of a new system, high code reuse and results in a higher quality of code.

This paper reports on a newly developed system to measure

Manuscript received September 19, 2005. This work was supported by the U.S. Department of Energy.

J. Nogiec, J. DiMarco, S. Kotelnikov, K. Trombly-Freytag, D. Walbridge, and M. Tartaglia are with the Fermi National Accelerator Laboratory, Batavia, Illinois 60510 USA (phone: 630-840-3081; fax: 630-840-2383; e-mail: nogiec@fnal.gov). the strength and alignment parameters (axes and angles) of accelerator magnets. The system applies the EMS software framework [1] and related methodology to the Single Stretched Wire measurement technique developed at Fermilab [4].

\section{Stretched-Wire Measurement Technique}

In the SSW system a wire is stretched throughout the magnet between two precision movement stages, with the return path of the wire loop running outside of the magnet or placed on the bottom of the beam pipe. The single stretched wire technique relies on accurate measurement of flux changes for given changes in position of the wire. Fig. 1 shows a schematic view of the wire stretched through a magnet.

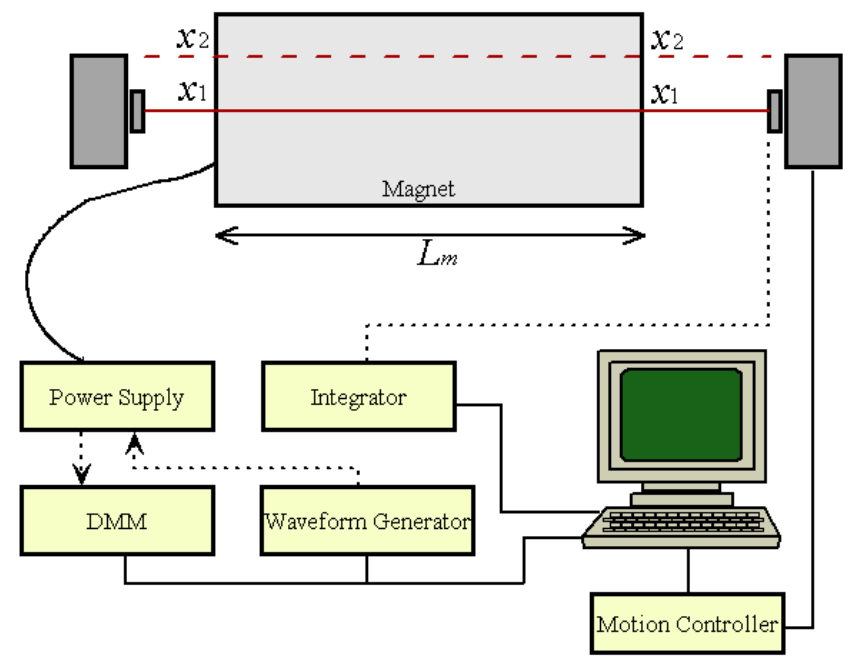

Fig. 1. SSW measurement system

Equation (1) defines the vector potential at the center of the quadrupole field, where $g$ is the gradient, $n, b_{n}, a_{n}$ the multipole order and normal and skew coefficients, and $R$ the reference radius. $x$ and $y$ are the horizontal and vertical positions in the frame of reference for the wire, while $x_{0}$ and $y_{0}$ are the offsets in the magnet coordinate frame.

$$
A_{z}=\Re\left[\sum_{n=1}^{\infty} g R \frac{\left(b_{n}+i a_{n}\right)}{n R^{n-1}}\left[\left(x-x_{0}\right)+i\left(y-y_{0}\right)\right]^{n}\right],
$$


As the wire is moved from point $\left(x_{1}, y_{1}\right)$ to point $\left(x_{2}, y_{2}\right)$, the change in flux is given by (2). The change in flux is only dependent on the starting and ending positions of the wire, not the path between them, and is given by

$$
\Phi=L_{m}\left[A_{z}\left(x_{2}, y_{2}\right)-A_{z}\left(x_{1}, y_{1}\right)\right]
$$

For large signals induced by DC magnet currents, alignment parameters can be directly determined by measuring flux change during wire motion. For weaker signals, such as those present during measurements of superconducting magnets at room temperatures, the flux change is induced in a motionless wire by powering the magnet with AC current. The Fourier analyzed flux changes determined at various wire positions are then combined to determine alignment parameters (see [4] for a detailed description).

\section{Software ARChiteCtURE}

A new SSW system has been built using the Extensible Measurement System (EMS) framework [1], [3], which is a reconfigurable data stream processing system [7]. Other systems built with the same framework include an integratorbased harmonics measurement system [2] and a DSP-based continuous measurement system [6]. The EMS framework is a component-based system developed in Java and $\mathrm{C} / \mathrm{C}++$, where data driven applications are assembled from components communicating via events. These components and their properties, as well as the flow of events (including data), are described in the system's configuration written in a specialized XML dialect. The visual configuration editor helps to develop configurations (Fig. 2), by visualizing event flows and component properties.

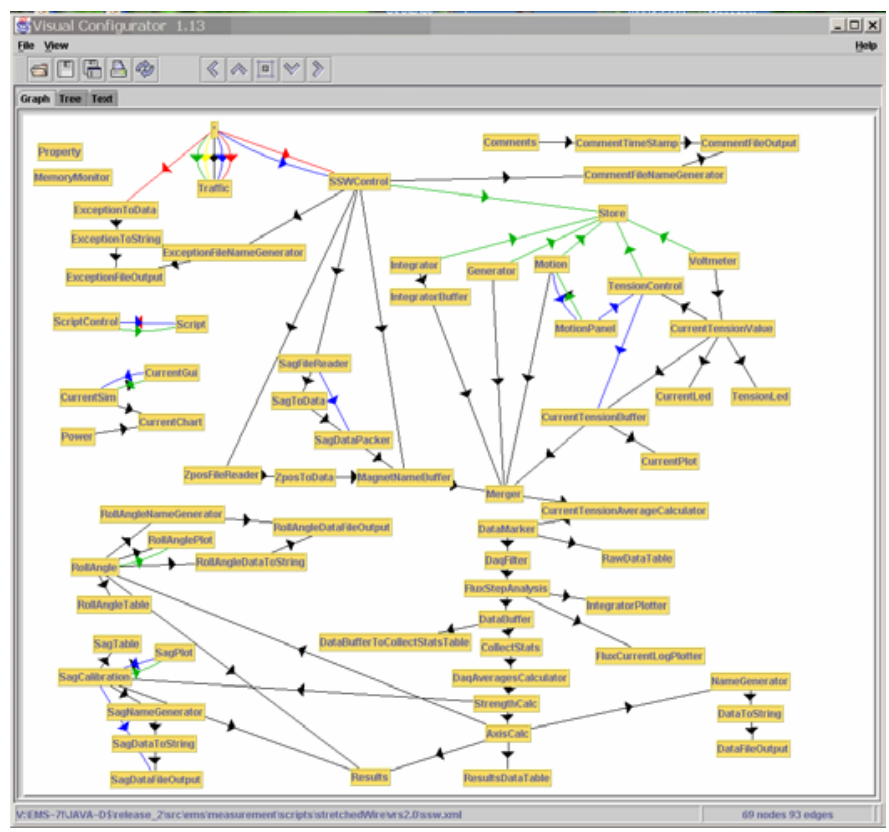

Fig. 2. Event flow in the SSW system.
The EMS framework allows for customization of the configured system with scripts. Scripts written in Jython, a Java-based implementation of Python, provide control over the measurement process. Scripts describe measurement sequences at a very high level of abstraction and supervise execution by sending control events to components, which acquire, process or visualize the data. Control events typically trigger operations on hardware, reconfigure components by applying a new set of properties (measurement parameters), or redirect data flow to appropriate processing components.

Measurement systems are assembled from a variety of prebuilt components. In addition to specialized hardware and analysis components specific to the SSW system, there exist many general-purpose EMS components, such as UI components and components implementing frequently used numerical algorithms (e.g. DSP or interpolation). Other components provide event flow control, system wide organizational functions and measurement monitoring. Diverse data archiving components provide persistence in an object-oriented database or implement saving to OS files in a variety of formats. Both exception and debugging notifications are managed within the system as events, allowing for flexible handling.
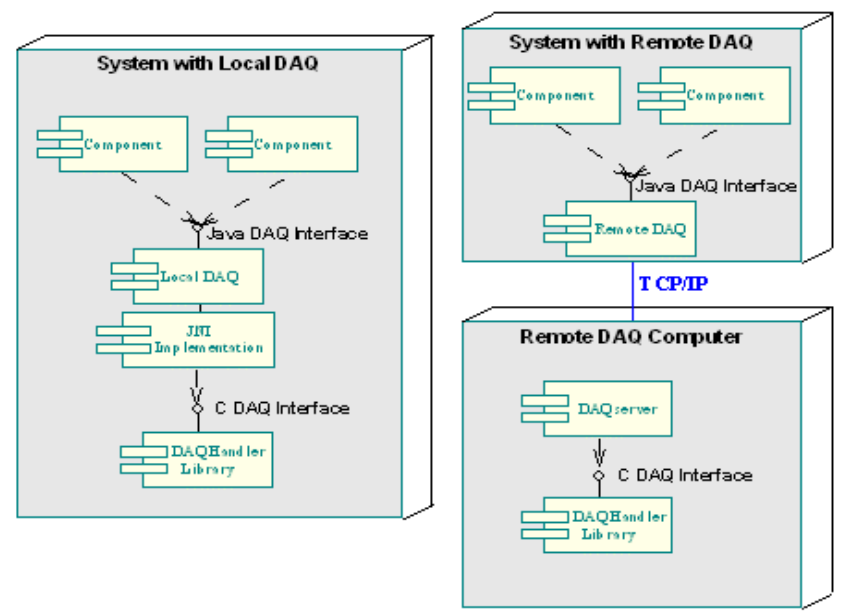

Fig. 3. DAQ Subsystem Organization.

\section{DAQ SUBSYSTEM}

The Data Acquisition Subsystem (DAQ) organizes access to instruments and devices via GPIB and VME buses. There exist two implementations for each of the supported hardware buses: a local implementation and a remote one (Fig 3). The local implementation employs Java Native Interface (JNI) to link Java with the $\mathrm{C}$ handler libraries that provide access to the buses via local interface cards (such as PCI cards for PCs or MXI cards for Sun workstations). The remote version employs components that serve as proxies for remotely implemented DAQ access. The remote DAQ consists of a server linked with the $\mathrm{C}$ handler libraries. In this solution, the Java and the $\mathrm{C}$ parts communicate via messages sent between sockets (TCP/IP) instead of the JNI system. 
All the DAQ software components can be configured to run in a simulation mode, in which they generate responses and do not attempt to access the actual hardware counterparts. All other parts of the system, including data visualization, data archival, and measurement scripting work exactly the same as with the hardware-enabled system. This significantly improves the ability of measurement developers to test various DAQ scenarios and analysis variants.

\section{INSTRUMENTATION}

The system is designed to accommodate various hardware configurations. It is possible to configure alternative versions of the same SSW measurement that employ different instruments, as long as replaced instruments are functionally equivalent. This allows for both easy comparison of alternative DAQ solutions and keeps the system operational in case of the limited availability of a specific instrument.

The system currently uses a voltmeter with a multiplexer to read tension and current (HP3457A or Keithley 2700), a precision voltage integrator to measure flux changes induced in the wire loop (Metrolab PDI5035), a motion controller to independently control and monitor movements of all stages (Newport ESP7000), a waveform generator to drive current for AC measurements (Agilent 33120A), a switch box to change from $\mathrm{AC}$ to $\mathrm{DC}$ measurement triggering modes (inhouse developed), and a power supply for magnet excitation during AC measurements (Kepco BOP 36-12M).

\section{MEASUREMENTS}

\section{A. System Setup}

Since the SSW system is fully configurable, its development lifecycle significantly differs from a typical software programming process. It is performed by a measurement designer, not a programmer, and includes the following steps:

1. Create a configuration by selecting and assembling components that control data flow, analyze data, provide partial and final data visualization, and archive data.

2. Prepare or modify measurement scripts, or update the measurement dictionaries, which contain arrays of movement sequences selectable at runtime.

3. Prepare or verify sets of parameters for each of the measurements.

4. Test and verify the correctness of the configuration and parameters by running in the simulation mode. Processing times and memory use can be examined during simulation.

The tested and validated system can then be handed to measurers for conducting measurements. During testing, selected sets of measurements can be set up to be executed either fully automatically or interactively, wherein the user is prompted for his/her choices of measurement dictionaries, repetitions, and other decisions. Each script can be repeated a requested number of times.

\section{B. Measurement Scripts}

Each measurement is controlled by a dedicated script and the system can be easily expanded by adding more scripts. The basic version has four main measurements: $D C, A C, S a g$, and Roll Angle as well as two auxiliary modes: Tension Adjustment and Monitoring.

1) $D C$ and $A C$ Measurement: The main measurements used to determine the true center axis of the magnetic field. Both DC and AC measurements are designed to run recursively, with each iteration beginning with the wire shifted to the field axis found in the previous iteration. An expected convergence helps to find the true field axis with a larger precision. The standard procedure for either type of measurement is to move both wire ends in the same ("co-directional" $C O$ ) or opposite ("counterdirectional" $C N$ ) directions along the $x$ and $y$ axes and concurrently measure flux, repeating as many times as needed. For each position, a value of current is measured. In the interactive mode, a user can limit measurements to any combination of $C O, C N$ and $x, y$. The analysis algorithm calculates the magnetic field fluxes at each wire position and then the average offset positions $\delta_{C O}$ and $\delta_{C N}$. Finally, using geometry and the sag calibration, a calculation of the magnet axes is performed. The final results are used to move the wire to the axes as defined by the offsets.

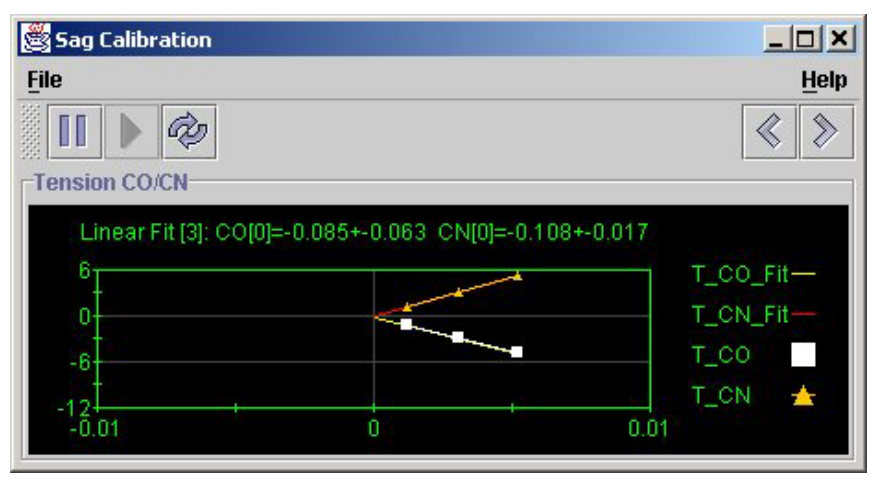

Fig. 4. Wire sag calibration window.

2) SAG Measurement: A calibration measurement that is used to correct for sag in the wire. The correction values are obtained by performing measurements while applying various tensions to the wire. Sag measurements are done when any system parameter has been changed (i.e., new wire, magnet, geometry, etc.). The calibration measurement usually performs $C O$ and $C N$ measurements in the vertical (y) direction in order to eliminate gravitational sag. Tension values are either taken from a file or provided by the user at each step. The script calculates offsets $\delta_{C O}$ and $\delta_{C N}$ for each tension and then makes a linear extrapolation to infinite tension in order to find the offset calibrations that would eliminate sag effects. A Sag Calibration window (Fig. 4) shows the 
plot of the resulting offsets versus inverse tension. In interactive mode, the results may be corrected manually by eliminating outlying points with a click of the mouse. A new approximation line is then calculated over the remaining points. Points may be removed or restored in this way until the best calibration performance is achieved. Finally, the result is saved for use in subsequent $D C$ and $A C$ measurements.

3) Roll Angle Measurement: A measurement of the roll angle of the magnet with respect to gravity. It measures the average offset $\delta_{C O}$ at a set of several different vertical positions taken from a file. In interactive mode, the user selects a vertical position for each step. The result is displayed as a plot of $\delta_{C O}$ function vs. twice the vertical position. The slope of the linear interpolation gives the roll angle value $\left(\theta_{\text {roll }}\right)$.

4) Monitoring Mode: A simple mode that enables the user to observe the status of the system by monitoring and displaying the motor statuses as well as values of the current and tension applied to the wire.

5) Tension Adjustment Mode: Another simple mode, which sets a specified tension to the wire using a tensionadjustment motor. The required tension value is taken from a parameter file or provided by the user interactively.

\section{On-Line Data Visualization}

The user interface consists of a number of windows selected at configuration time (see Fig. 5). Because the system has the ability to adapt its presentation to the current need, each different measurement will present its own set of display and controlling windows. Many aspects of these displays are also alterable at runtime (e.g., colors, selected data items, titles, positions, sizes, etc.).

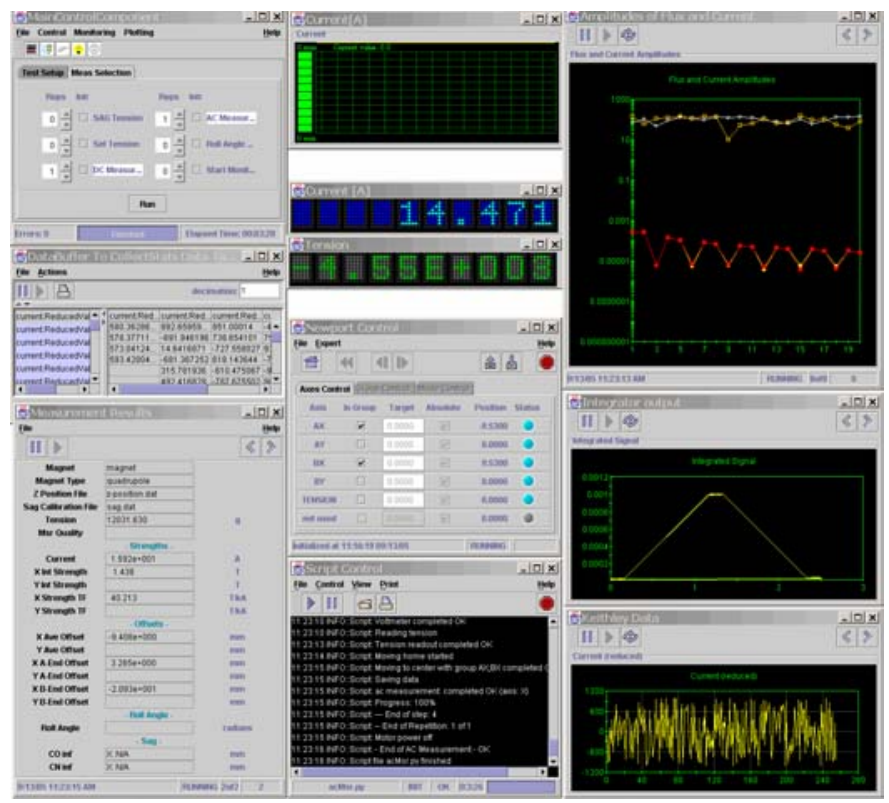

Fig. 5. SSW system user interface.
All or selected parts of the raw data and the analysis results can be both displayed in real-time and/or saved for later perusal. That includes intermediate data from any chosen point in the data processing flow, which is at the output of any component.

Graphical and numerical data displays are universal components and are capable of displaying any selected data element in the system. They are equipped with a memory feature that also allows reviewing data from previous data acquisitions and processing steps. They include the possibility to freeze an interesting display without stopping or pausing the rest of the system. This allows for closer examination of particular data. All display components have an option to produce a hardcopy of their contents.

\section{CONCLUSION}

The described SSW system is part of the family of measurement systems, a group of systems built based on a common software framework. The system is configured from general-purpose and specialized components. It allows for runtime monitoring and tailoring of almost all the details of its functioning. Measurement algorithms are in the form of scripts that can be easily modified and added to the system. Portability of the system stems from the fact that it is mostly written in Java with a minimal platform-dependent code necessary to interface to instrumentation buses. Although the system can work completely autonomously and even be scheduled to run in the background, its major strengths lay in its extensibility, flexibility and openness. These features make it especially attractive for the $\mathrm{R} \& \mathrm{D}$ type measurements and in the environments where measurement subjects and requirements change frequently. Several configurations of the system have been already explored and used, with computers running under the Linux, Solaris and VxWorks operating systems.

\section{REFERENCES}

[1] J.M. Nogiec, J. Sim, K. Trombly-Freytag, D. Walbridge, "EMS: A Framework for Data Acquisition and Analysis," ACAT2000, Batavia, 2000.

[2] J.M. Nogiec et al., "A Flexible and Configurable System to Test Accelerator Magnets," PAC'01, Chicago, 2001.

[3] J.M. Nogiec, E. Desavouret, S. Kotelnikov, K. Trombly-Freytag, and D. Walbridge, "Configuring Systems from Components: The EMS Approach," ACAT'03, Tsukuba, 2003.

[4] J. DiMarco, H. Glass, M. Lamm, P. Schlabach, C. Sylvester, J. Tompkins, J. Krzywinski, "Field Alignment of Quadrupole Magnets for the LHC Interaction Regions", MT-16, Ponte Vedra Beach, Florida, 1999.

[5] H. Brueck et al., "Magnetic Measurements of a Superferric Quadrupole for the Tesla Test facility with a Stretched Wire and AC Current," MT15, Beijing, China, 1997.

[6] G. Velev et al., "A Fast Continuous Magnetic Field Measurement System Based on Digital Signal Processors," MT-19, Genova, Italy, 2005, submitted for publication

[7] J. Nogiec, K. Trombly-Freytag, "A Dynamically Reconfigurable Data Stream Processing System,” CHEP’04, Interlaken, Switzerland, 2004. 